\title{
Psychiatric outcome at least 20 years after trauma: A survey on the status of subjective general health and psychiatric symptoms with a focus on posttraumatic stress disorder
}

Halvachizadeh, Sascha ; Teuber, Henrik ; Allemann, Florin ; Luidl, Anna Theresa ; von Känel, Roland ; Zelle, Boris ; Tiziani, Simon ; Rauen, Katrin ; Pape, Hans-Christoph ; Pfeifer, Roman

\begin{abstract}
BACKGROUND Information on long-term psychiatric sequelae after severe trauma is sparse. We therefore performed a survey addressing several symptoms related to post traumatic stress disorder (PTSD) in patients that sustained multiple injuries more than 20 years after trauma. METHODS Patients injured between January 1, 1973 and December 31, 1990 were contacted at least 20 years later. We included multiply injured patients aged between 3 and 60 years of age from a single level I Trauma center. A questionnaire based on the Diagnostic and Statistical Manual of Mental Disorders (DSM-IV) diagnostic criteria for PTSD, including individual symptoms related to intrusion, avoidance, and hyperarousal was sent to all patients. RESULTS 359 (56.35\%) patients received a questionnaire. Out of these, 337 (93.87\%) returned the questionnaire and were included in the study (223 males (66.17\%) and 114 females $(33.82 \%)$ ). Mean follow-up was $29.5 \pm 8.5$ years. Nearly half the study population $(47.18 \%)$ experienced lasting psychiatric sequelae, such as intrusive recollection $(\mathrm{n}=65,19.28 \%)$, avoidance $(\mathrm{n}=92,27.29 \%)$, or hyperarousal $(\mathrm{n}=95,28.18 \%)$ at least monthly. Ten patients $(2.96 \%)$ fulfilled all DSM-IV diagnostic criteria for PTSD. A total of 131 (38.87\%) patients reported fair or poor general health status. There was no difference in injury severity in patients with or without PTSD (Injury Severity Score (ISS) 18.33 vs. 20.36, respectively, $\mathrm{p}=0.52$ ) or PTSD-related symptoms including intrusion (19.88 vs. 20.32, $\mathrm{p}=0.74$ ), avoidance (19.99 vs. $20.3, \mathrm{p}=0.79$ ) and hyperarousal (19.36 vs. $20.68, \mathrm{p}=0.26$ ). CONCLUSION At least 20 years after injury, no correlation was found between the development of psychiatric complications and the severity of injury. While the rate of full-blown PTSD was low, nearly half the study population regularly suffered from at least one psychiatric symptom attributable to the initial trauma. Awareness for the development of psychiatric complications and early initiation of psychiatric counseling is advisable. LEVEL OF EVIDENCE Level II, Prognostic and Epidemiologic.
\end{abstract}

DOI: https://doi.org/10.1097/TA.0000000000002232

Posted at the Zurich Open Repository and Archive, University of Zurich

ZORA URL: https://doi.org/10.5167/uzh-168715

Journal Article

Accepted Version

Originally published at:

Halvachizadeh, Sascha; Teuber, Henrik; Allemann, Florin; Luidl, Anna Theresa; von Känel, Roland; Zelle, Boris; Tiziani, Simon; Rauen, Katrin; Pape, Hans-Christoph; Pfeifer, Roman (2019). Psychiatric outcome at least 20 years after trauma: A survey on the status of subjective general health and psychiatric 
symptoms with a focus on posttraumatic stress disorder. Journal of Trauma and Acute Care Surgery, 86(6):1027-1032.

DOI: https://doi.org/10.1097/TA.0000000000002232 


\section{Psychiatric outcome at least 20 years after trauma: A survey on the status}

\section{of subjective general health and psychiatric symptoms with a focus on}

\section{posttraumatic stress disorder}

Sascha Halvachizadeh, MD ${ }^{1}$, Henrik Teuber, DO ${ }^{1}$, Florin Allemann, $\mathrm{MD}^{1}$, Anna Theresa Luidl, MD, Roland von Känel, $\mathrm{MD}^{2}$, Boris Zelle, $\mathrm{MD}^{3}$, Simon Tiziani, MD ${ }^{1}$, Katrin Rauen, $\mathrm{MD}^{4}$, Hans-Christoph Pape, MD, FACS ${ }^{1}$, Roman Pfeifer, MD, FEBS ${ }^{1}$

${ }^{1}$ Department of Trauma, University Hospital Zurich, University of Zurich, Switzerland, Rämistrasse 100, 8091 Zurich

${ }^{2}$ Department of Consultation-Liaison Psychiatry and Psychosomatic Medicine, University Hospital Zurich, University of Zurich, Switzerland, Culmannstrasse 8, 8091 Zurich

${ }^{3}$ University of Texas Health Science Center at San Antonio, 8300 Floyd Curl Drive, San Antonio, TX 78229 USA

${ }^{4}$ Institute for Regenerative Medicine, University of Zurich, Switzerland, Wagistrasse 12, 8952 Schlieren

\section{Corresponding author}

Sascha Halvachizadeh

University Hospital Zurich

Department of Trauma

Rämistrasse 100

8091 Zurich

Sascha.halvachizadeh@usz.ch

Tel.: +41-44-2551111

Mobile: +41-78-7598859

Fax: +41-44-2554466 


\section{Conflict of interest statement}

None of the authors have any conflicts of interests to declare. The authors received no pharmaceutical or industrial support for this study. No further direct or indirect financial support or other assets were transferred to the authors or their family members for this study.

\section{Presentation}

These results were presented in the $77^{\text {th }}$ Annual Meeting of AAST and Clinical Congress of Acute Care Surgery and $4^{\text {th }}$ World Trauma Congress; WTC Oral Presentation for AAST20180021.

\section{Funding}

For the present 20-year follow-up, institutional funding was used. No further funding sources were utilized. 


\section{Background}

Information on long-term psychiatric sequelae after severe trauma is sparse. We therefore performed a survey addressing several symptoms related to post traumatic stress disorder (PTSD) in patients that sustained multiple injuries more than 20 years after trauma.

\section{Methods}

Patients injured between January 1, 1973 and December 31, 1990 were contacted at least 20 years later. We included multiply injured patients aged between 3 and 60 years of age from a

single level I Trauma center. A questionnaire based on the Diagnostic and Statistical Manual of Mental Disorders (DSM-IV) diagnostic criteria for PTSD, including individual symptoms related to intrusion, avoidance, and hyperarousal was sent to all patients.

\section{Results}

$359(56.35 \%)$ patients received a questionnaire. Out of these, $337(93.87 \%)$ returned the questionnaire and were included in the study (223 males (66.17\%) and 114 females (33.82\%)). Mean follow-up was $29.5 \pm 8.5$ years. Nearly half the study population $(47.18 \%)$ experienced lasting psychiatric sequelae, such as intrusive recollection $(n=65,19.28 \%)$, avoidance $(n=92,27.29 \%)$, or hyperarousal $(n=95,28.18 \%)$ at least monthly. Ten patients (2.96\%) fulfilled all DSM-IV diagnostic criteria for PTSD. A total of 131 (38.87\%) patients reported fair or poor general health status. There was no difference in injury severity in patients with or without PTSD (Injury Severity Score (ISS) 18.33 vs. 20.36, respectively, p = 0.52) or PTSD-related symptoms including intrusion (19.88 vs. 20.32, $\mathrm{p}=0.74$ ), avoidance (19.99 vs. $20.3, \mathrm{p}=0.79$ ) and hyperarousal (19.36 vs. $20.68, \mathrm{p}=0.26)$.

\section{Conclusion}

At least 20 years after injury, no correlation was found between the development of psychiatric complications and the severity of injury. While the rate of full-blown PTSD was low, nearly half the study population regularly suffered from at least one psychiatric 
symptom attributable to the initial trauma. Awareness for the development of psychiatric complications and early initiation of psychiatric counseling is advisable.

\section{Level of Evidence}

Level II, Prognostic and Epidemiologic

\section{Keywords}

PTSD, Long-term follow-up, severe injury, multiple trauma, psychiatric complication, intrusion, avoidance, arousal 


\section{Background}

After severe trauma, patients may suffer from psychological, psychiatric, cognitive and/or behavioral disabilities (1). Severely injured patients are prone to develop psychiatric diseases and mental disorders with higher rates of suicide or additional traumatic events when compared to the general population $(2,3)$. In addition, psychiatric disorders, such as depression, anxiety or posttraumatic stress disorder (PTSD) may interfere with the physical rehabilitation process in these patient groups. Patients who exhibited a higher sense of selfefficacy showed improved outcomes and rehabilitation results (4). Finally, family involvement in the rehabilitation process also appears to show positive effects on outcome (5). Our group has previously performed a 10-year follow-up survey to assess long-term psychological and general quality of life (6). The survey confirmed that patients with injuries to the lower extremities or intra-articular fractures were significantly more likely to experience poor outcomes $(7,8)$. Moreover, traumatic brain injuries and spinal cord injuries were independent predictors of long-term disability $(2,9,10)$.

The aim of the current 20-year follow-up study was to answer the following questions:

1. Is the severity of multiple injured patients associated with the development of PTSD or PTSD-related symptoms, such as intrusion, avoidance or arousal?

2. Are psychiatric problems and subjective perception of general health associated with injury distribution or injury severity?

\section{Methods}

\section{Ethical considerations}

This study was conducted according to the declaration of Helsinki (11). The local Ethic

Committee approved this study (Ethical Committee Trial ID-Number 2325-200/03/22). Participants who consented to participate in this study were included. 


\section{Study Population}

In our previous studies $(12,13)$ a database was established to allow for long-term follow-up. Utilizing an exhaustive recruitment process with multiple steps to find patients that had moved $(6,14)$, patients treated between January 1, 1973 and December 31, 1990 at a single level 1 trauma center were contacted. All patients were contacted by phone or mail prior to sending out the questionnaire. With a returned questionnaire, patients consented to participate and were included in this study. This investigation was designed as a prospective cohort study. All multiply injured patients between 3-60 years of age with a minimum follow-up of 20 years and a properly completed questionnaire fulfilled the study inclusion criteria. Inability to fill out the questionnaire as well as incomplete data lead to exclusion.

\section{Questionnaire}

The self-administered questionnaire consisted of 118 questions. Questions assessing PTSD were strictly based on DSM-IV criteria for PTSD (15). The questionnaire investigated psychiatric symptoms, the ability to complete activities of daily living, work status, and the degree of current psychotherapeutic treatment or rehabilitation. General health status was assessed through several self-ratings with scores ranging from one ("poor") to five (“excellent”).

\section{Definitions of PTSD}

Questions $(n=33)$ assessing PTSD were based on the American Psychiatric Association (APA) DSM-IV criteria for PTSD. Most of the questions $(n=23)$ were adopted from the Impact of Event Scale (IES) $(16,17)$. According to the DSM-IV, six categories of symptoms (A-F) must be evaluated to diagnose PTSD. Category A establishes the occurrence of a lifethreatening traumatic event. Categories B-D were evaluated with the following number of questions (n): Category $B$ evaluates re-experiencing the trauma, recurrent and stressful 
memories, flashbacks, nightmares or intrusion $(\mathrm{n}=8)$; Category $\mathrm{C}$, the symptoms of avoidance $(n=9)$, and Category $D$ evaluates the presence of arousal $(n=7)$. Specifically, the questionnaire evaluated trauma-related psychiatric complications on a 4-point numerical scale assessing how often patients experienced these symptoms within the last month:

1) Never: 1 point

2) Seldom (once or twice per month): 2 points

3) Occasionally (once or twice per week): 3 points

4) Often (at least once per day): 4 points

The PTSD criteria for each category were fulfilled, if a score of greater than 3 points was tabulated. Criteria for Category $E$ were fulfilled if the above symptoms were present for more than one month. Category $F$ assessed whether the above disturbances have led to clinically significant distress or impairment in social or occupational situations. The PTSD portion of the questionnaire was based exclusively on these diagnostic categories and therefore reliably suggests the presence or absence of PTSD. Patients were then stratified into two groups: those who fulfilled the above diagnostic criteria for PTSD as well as those experiencing at least one item of the intrusion, avoidance or arousal symptom cluster and those who did not.

\section{Statistics}

Statistical analysis was performed using GraphPad Prism® statistical software (Version 7.00 for Windows, GraphPad Software, La Jolla California USA, www.graphpad.com) as well as SPSS Statistics program (IBM Corp. Released 2017. IBM SPSS Statistics for Windows, Version 25.0. Armonk, NY: IBM Corp.). The data was tested for normal distribution using the Kolmogorov-Smirnov test. The ANOVA test was used to compare groups on continuously scaled variables with a normal distribution. The Kruskal Wallis test was used for non-normal distributed continuous variables with a skewed distribution. The Pearson $\chi^{2}-$ 
test was applied to compare groups on categorical variables. Significance level was set at a pvalue $<0.05$ (two-tailed).

\section{Results}

Patient selection and demographics

Figure 1 shows the flow diagram of patient enrollment. Out of 637 patients that were initially enrolled (2), 36 (5.6\%) patients had died and 242 (38.0\%) were lost to follow-up. Of the 359 patients who received the questionnaire, 337 (93.9\%) responded, 21 (5.8\%) did not respond, and one patient $(0.3 \%)$ returned an illegible questionnaire. The demographics are summarized in Table 1. Patients who did not contribute to this study demonstrated a similar demographic distribution (78\% male, mean age at the time of injury of $26.6 \pm 12.8$ years and ISS of $21 \pm$ 10 points). We found no significant differences between the population that was included in this study and those who did not contribute regarding their demographics.

\section{General outcome}

General health was reported as excellent $(n=14,4.1 \%)$, very good $(n=43,12.8 \%)$, good $(n=149,44.2 \%)$, fair $(n=107,31.8 \%)$ or poor $(n=24,7.1 \%)$. More than one third of patients $(38.87 \%)$ rated their current health status as fair or poor, whereas $16.91 \%$ of patients reported excellent or very good health.

With respect to physical activity, 10 patients (2.9\%) reported no capability of being physically active, 118 patients (35.0\%) claimed severe limitations, 111 (32.9\%) had few limitations, $46(13.6 \%)$ minimal, and $52(15.4 \%)$ had no limitations during physical activity.

More than one in four patients $(n=88,26.1 \%)$ experienced at least one additional psychiatric insult through further trauma or other traumatic events with $53(15.7 \%)$ patients suffering additional severe injuries or experiencing fires or explosions. Thirty-four patients $(10.0 \%)$ had to cope with life-threatening disease and $31(9.2 \%)$ became victims of violence. 


\section{PTSD}

\section{PTSD Criterion A: Traumatic event}

All patients suffered multiple injuries at least 20 years prior to the study. Average ISS was $20.3 \pm 9.2$. Sustaining multiple trauma, our patients suffered life-threatening injuries and criteria A for PTSD diagnosis was therefore considered fulfilled.

\section{PTSD Criterion B: Intrusion}

Nearly one in four patients $(n=65 / 337,19.3 \%)$ recollected past intrusive symptoms of their trauma at least once per month. Out of these sixty-five patients, sixty patients $(92.3 \%)$ reported intrusion either occasionally $(n=41,63.1 \%)$ or often $(n=19,29.2 \%)$. The remaining five patients $(7.7 \%)$ rarely experienced these symptoms.

\section{PTSD Criterion C: Avoidance}

Avoidance was reported in $92 / 337$ patients $(27.4 \%)$, out of which 21 patients $(22.8 \%)$ frequently experienced symptoms of avoidance, 32 of them (34.8\%) occasionally and 39 of them $(42.4 \%)$ rarely.

\section{PTSD Criterion D: Hyperarousal}

With respect to criteria $\mathrm{D}, 95 / 337$ (28.2\%) patients suffered from hyperarousal at least once per month. Out of these, 60 patients (63.2\%) experienced symptoms of arousal either weekly $(n=40,42.1 \%)$, or daily $(n=20,21.1 \%)$. Thirty-five patients $(36.8 \%)$ reported to suffer rarely from hyperarousal.

\section{PTSD diagnosis (all symptom dimensions)}

In this study, $159(47.2 \%)$ patients reported symptoms in at least one item of the intrusion, avoidance or arousal cluster. Ten patients $(2.9 \%)$ reported suffering daily symptoms in all three clusters and may therefore fulfill the DSM IV diagnostic criteria of PTSD. Patients with PTSD did not significantly differ in age, gender, ISS, and Maximum Abbreviated Injury Scale (MAIS) from those not fulfilling PTSD criteria. Further, patients suffering any PTSDrelated symptoms at least once per month also did not differ demographically to those with 
no PTSD-related symptoms in all categories. The development of psychiatric complications was significantly associated with psychiatric treatment $(\mathrm{p}<0.01)$. Psychiatric treatment after trauma was common in all patient groups. Unemployment due to the initial trauma was significantly more likely in patients with PTSD $(\mathrm{p}=0.009)$ and those reporting symptoms of hyperarousal $(\mathrm{p}=0.04)$. Additional trauma or early retirement was not more commonly seen in those patients with PTSD or PTSD-related symptoms. These findings are summarized in Table 2.

\section{Discussion}

Traumatic injuries continue to be among the leading causes of death and disability worldwide (18). In patients surviving severe trauma, psychiatric sequelae are frequent and may lead to long term disability unrelated to their medical condition (19).

This study shows a low prevalence of full blown PTSD (3\%), comparable to the life-time prevalence of PTSD in the general population (30). In contrast, nearly half of the patients suffer at least one symptom of PTSD (i.e. intrusion, avoidance, or hyperarousal) after more than 20 years after their initial injury. No association with the development PTSD or PTSDrelated symptoms was observed, neither was there an association with their self-rated general health status and the injury severity or distribution.

We feel that our study has both limitations and advantages. One might argue, that the management of severely injured patients may differ from that of other institutions. However, a single center survey reduces the likelihood of inter-hospital differences in trauma management. The questionnaires were completed by the patients, and represent a subjective self-evaluation. Inability of some patients to successfully fill out the questionnaire due to older age or long-term trauma sequelae may be considered another confounding variable. However, since $93 \%$ of patients returned a properly completed questionnaire, we feel that this did not negatively affect our results. In addition, questions in our questionnaire derived 
exclusively from validated questionnaires. Furthermore, the distinct possibility exists that some patients who did not consent to participate, or did not return the questionnaire, are unwilling to discuss and relive the psychiatric stress of their respective trauma. This may have caused under-reporting of a significant level of patients suffering from long-term psychiatric complications, which cannot be completely ruled out. Moreover, when the complete syndrome of PTSD is regarded as an isolated outcome measure, the statistical power may have been too low to identify significant differences ( $n=10$ out of 337$)$.

Finally, we could not evaluate the psychiatric influence that subsequent traumatic events experienced by our patient population had on study results.

In contrast, the questionnaire was designed, and patients were specifically instructed to focus their assessment on the initial traumatic event. We feel that further strengths of this study were the high inclusion rate of greater than $90 \%$ and the large degree of long-term follow-up data, with this data reflecting the patients' current state of health and mind.

To our knowledge, this assessment of the psychiatric complications of multiple injured patients after more than 20 years represent the longest follow-up in the literature, thus justifying our conclusions.

In this long-term follow-up study, a high percentage of patients still reported unsatisfactory general health. This is in accordance with previous studies that evaluated multiple factors including inability to return to work and socio-economic factors $(12,20)$. We concur with previous authors that these issues persist and do not appear to regress over time (21).

Severe injury is not necessarily sufficient to explain the development of PTSD (22). Risk factors for developing PTSD include the extent to which the traumatic event remains in the patient's memory (23), pre-trauma vulnerability (24), the magnitude of the stressor, anticipation of the event, immediate reactions to the trauma and post-trauma factors (25), as well as peri-traumatic dissociation (26). In the literature, PTSD, depression and other 
psychiatric disorders may be a cause of the trauma itself through self-inflicted injuries and attempted suicide $(3,27)$. The present analysis of our data showed that less than $3 \%$ of patients fulfilled diagnostic criteria of PTSD more than 20 years after trauma. A wide range in the life-time prevalence of PTSD after trauma has been shown in the literature (28). This is most likely due to high variability in study design and utilization of different diagnostic criteria for PTSD (e.g. ICD-10 and DSM-5). Our long-term results of PTSD prevalence are in line with the literature, with several studies observing PTSD prevalence between $2-8 \%$ (2932). In some studies, higher levels of PTSD in the short-term have been observed. One recent one to two year follow-up survey of 455 patients reported that $22 \%$ of patients suffered from PTSD and 24\% suffered from decreased quality of life (33).

Higher rates of PTSD have been reported in soldiers with $15 \%$ of Vietnam War and $12 \%$ of Gulf War veterans still suffering from PTSD in a study published in 2011 (34). The World Health Organization's (WHO) world mental health surveys in patients who suffered traffic accidents also support our data having shown overall prevalence of PTSD after lifethreatening motor vehicle collisions of $2.5 \%$ (35).

Although the prevalence of PTSD in our study population is comparable to the life-time prevalence, our data show that nearly half of patients suffering from PTSD-related symptoms at least once per month. According to this survey, these symptoms likely to be related with the initial injury, the patients suffered more than 20 years ago. It has been recently shown that the likelihood for developing these symptoms depends on the type of initial trauma (intimate and non-intimate interpersonal) (36). The type of trauma was stratified in intimate and nonintimate interpersonal trauma. PTSD related symptoms were more likely in patients who suffered intimate interpersonal trauma (36). The prevalence of PTSD bases on the simultaneous presence of all PTSD-related symptoms, yet, each individual symptom may influence the patients general quality of life. Notwithstanding a decreased diagnostic rate of 
PTSD, the threshold for psychiatric consultation should be chosen appropriately according to the individual symptoms.

\section{Conclusion}

More than two decades after severe injury, a considerable number of patients were not satisfied with their physical and mental health, although no correlation between poor health and injury severity or distribution was found. While general incidence of PTSD was low, nearly half of the study population frequently reported at least some PTSD related symptoms. These symptoms as well as PTSD did not correlate with ISS or MAIS. We feel that the threshold for psychiatric counseling after trauma should be low when considering the high long-term prevalence of post-traumatic psychiatric symptoms. 


\section{Author contributions}

Sascha Halvachizadeh: Writing and revision of the manuscript, analysis, interpretation of data and statistics, searched literature

Henrik Teuber: Interpretation of data, critical revision of the manuscript, searched literature Florin Allemann: Critical revision of the manuscript

Anna Theresa Luidl: Data collection; her doctoral thesis was part of this study

Roland von Känel: Critical revision of the manuscript

Boris Zelle: Critical revision the manuscript

Simon Tiziani: Critical revision of the manuscript

Katrin Rauen: Critical revision of the manuscript

Hans-Christoph Pape: Interpretation of data and statistics, critical revision of the manuscript, establishment of the primary databank, development of study

Roman Pfeifer: Analysis and interpretation of data, statistics, critical revision of the manuscript, data collection, development of the study, searched literature

All authors read and approved the final manuscript.

\section{Conflict of interest statement}

None of the authors have any conflicts of interests to declare. The authors received no pharmaceutical or industrial support for this study. No further direct or indirect financial support or other assets were transferred to the authors or their family members for this study.

\section{Funding}

For the present 20-year follow-up, institutional funding was used. No further funding sources were utilized. 


\section{References}

1. Thurston RJ. Brain injury, memory and learning: University of Calgary; 1999.

2. Steel J, Youssef M, Pfeifer R, Ramirez JM, Probst C, Sellei R, Zelle BA, Sittaro N-A, Khalifa F, Pape HC. Health-related quality of life in patients with multiple injuries and traumatic brain injury 10+ years postinjury. J Trauma Acute Care Surg. 2010;69(3):523-31.

3. Probst C, Zelle BA, Sittaro NA, Lohse R, Krettek C, Pape HC. Late death after multiple severe trauma: when does it occur and what are the causes? J Trauma Acute Care Surg. 2009;66(4):1212-7.

4. Anke AG, Fugl-Meyer AR. Life satisfaction several years after severe multiple trauma-a retrospective investigation. Clin Rehabil. 2003;17(4):431-42.

5. Hanks RA, Rapport LJ, Vangel S. Caregiving appraisal after traumatic brain injury: The effects of functional status, coping style, social support and family functioning. NeuroRehabilitation. 2007;22(1):43-52.

6. Pape H-C, Zelle B, Lohse R, Hildebrand F, Krettek C, Panzica M, Duhme V, Sittaro N. Evaluation and outcome of patients after polytrauma - can patients be recruited for longterm follow-up? Injury. 2006;37(12):1197-203.

7. Dienstknecht T, Pfeifer R, Horst K, Sellei R, Berner A, Zelle B, Probst C, Pape H. The long-term clinical outcome after pelvic ring injuries. Bone Joint J. 2013;95(4):548-53.

8. Zelle B, Brown S, Panzica M, Lohse R, Sittaro N, Krettek C, Pape H. The impact of injuries below the knee joint on the long-term functional outcome following polytrauma. Injury. 2005;36(1):169-77.

9. Pape HC, Probst C, Lohse R, Zelle BA, Panzica M, Stalp M, Steel JL, Duhme HM, Pfeifer R, Krettek C, et al. Predictors of late clinical outcome following orthopedic injuries after multiple trauma. J Trauma. 2010;69(5):1243-51. 
10. Rauen K, Reichelt L, Schaepers B, Müller F, Jahn K, Plesnila NJBi. Crocflamechronic neuroinflammation and its role for cognitive decline and long-term sequels following traumatic brain injury. 2017(43287):890-.

11. Jama WMAJ. World Medical Association Declaration of Helsinki: ethical principles for medical research involving human subjects. 2013;310(20):2191.

12. Pfeifer R, Lichte P, Zelle BA, Sittaro N-A, Zilkens A, Kaneshige JR, Pape H-C. Socio-economic outcome after blunt orthopaedic trauma: Implications on injury prevention. Patient Saf Surg. 2011;5(1):9.

13. Falkenberg L, Zeckey C, Mommsen P, Winkelmann M, Zelle BA, Panzica M, Pape H-C, Krettek C, Probst C. Long-term outcome in 324 polytrauma patients: what factors are associated with posttraumatic stress disorder and depressive disorder symptoms? Eur J Med Res. 2017;22(1):44.

14. Sittaro NA, Lohse R, Panzica M, Probst C, Pape HC, Krettek C. Hannoverpolytrauma-longterm-study HPLS. Versicherungsmedizin/herausgegeben von Verband der Lebensversicherungs-Unternehmen eV und Verband der Privaten Krankenversicherung eV. 2007;59(1):20-5.

15. Yehuda R. Post-traumatic stress disorder. N Engl J Med. 2002;346(2):108-14.

16. Horowitz M, Wilner N, Alvarez W. Impact of Event Scale: a measure of subjective stress. Psychosom Med. 1979;41(3):209-18.

17. Maercker A, Schützwohl M. Erfassung von psychischen Belastungsfolgen: Die Impact of Event Skala-revidierte Version (IES-R). Diagnostica. 1998.

18. Krug EG, Sharma GK, Lozano R. The global burden of injuries. Am J Public Health. 2000;90(4):523.

19. Burri A, Maercker A. Differences in prevalence rates of PTSD in various European countries explained by war exposure, other trauma and cultural value orientation. BMC Res Notes. 2014;7:407. 
20. Michaels AJ, Michaels CE, Smith JS, Moon CH, Peterson C, Long WB. Outcome from injury: general health, work status, and satisfaction 12 months after trauma. J Trauma Acute Care Surg. 2000;48(5):841-50.

21. van Oostrom SH, Gijsen R, Stirbu I, Korevaar JC, Schellevis FG, Picavet HSJ, Hoeymans N. Time trends in prevalence of chronic diseases and multimorbidity not only due to aging: data from general practices and health surveys. PloS one. 2016;11(8):e0160264.

22. Herrera-Escobar JP, Al Rafai SS, Seshadri AJ, Weed C, Apoj M, Harlow A, Brasel K, Kasotakis G, Kaafarani HMA, Velmahos G, et al. A multicenter study of post-traumatic stress disorder after injury: Mechanism matters more than injury severity. Surgery. 2018.

23. Caspi Y, Gil S, Ben-Ari IZ, Koren D, Aaron-Peretz J, Klein E. Memory of the traumatic event is associated with increased risk for PTSD: A retrospective study of patients with traumatic brain injury. J Loss Trauma. 2005;10(4):319-35.

24. Ozer EJ, Best SR, Lipsey TL, Weiss DS. Predictors of posttraumatic stress disorder and symptoms in adults: a meta-analysis. Psychol Bull. 2003;129(1):52.

25. Shalev AY, Peri T, Canetti L, Schreiber S. Predictors of PTSD in injured trauma survivors: a prospective study. Am J Psychiatry. 1996;153(2):219.

26. Ursano RJ, Fullerton CS, Epstein RS, Crowley B, Kao T-C, Vance K, Craig KJ, Dougall AL, Baum A. Acute and chronic posttraumatic stress disorder in motor vehicle accident victims. Am J Psychiatry. 1999;156(4):589-95.

27. Poole GV, Lewis JL, Devidas M, Hauser CJ, Martin RW, Thomae KR. Psychopathologic risk factors for intentional and nonintentional injury. J Trauma Acute Care Surg. 1997;42(4):711-5.

28. Ellis J, Zaretsky A. Assessment and management of posttraumatic stress disorder. Continuum (Minneap Minn). 2018;24(3):873-92.

29. Roberts AL, Gilman SE, Breslau J, Breslau N, Koenen KCJPm. Race/ethnic differences in exposure to traumatic events, development of post-traumatic stress disorder, 
and treatment-seeking for post-traumatic stress disorder in the United States. 2011;41(1):7183.

30. Breslau N. Epidemiology of posttraumatic stress disorder in adults. The Oxford Handbook of Traumatic Stress Disorders Oxford University Press: New York. 2012:84-97.

31. Pape JC, Binder EB. [Psychological trauma as risk for delayed psychiatric disorders: epigenetic mechanisms]. Nervenarzt. 2014;85(11):1382-9.

32. Perrin M, Vandeleur CL, Castelao E, Rothen S, Glaus J, Vollenweider P, Preisig M. Determinants of the development of post-traumatic stress disorder, in the general population. Social psychiatry and psychiatric epidemiology. 2014;49(3):447-57.

33. Danielsson F, Larsen MS, Nørgaard B, Lauritsen J. Quality of life and level of posttraumatic stress disorder among trauma patients: A comparative study between a regional and a university hospital. Scand J Trauma Resusc Emerg Med. 2018;26(1):44.

34. Javidi H, Yadollahie M. Post-traumatic stress disorder. Int J Occup Environ Med 2011;3(1 January).

35. Stein DJ, Karam EG, Shahly V, Hill ED, King A, Petukhova M, Atwoli L, Bromet EJ, Florescu S, Haro JM. Post-traumatic stress disorder associated with life-threatening motor vehicle collisions in the WHO World Mental Health Surveys. BMC psychiatry. 2016;16(1):257.

36. Forbes D, Lockwood E, Phelps A, Wade D, Creamer M, Bryant RA, McFarlane A, Silove D, Rees S, Chapman C, et al. Trauma at the hands of another: distinguishing PTSD patterns following intimate and nonintimate interpersonal and noninterpersonal trauma in a nationally representative sample. J Clin Psychiatry. 2014;75(2):147-53. 
$\mathrm{n}=637$

(Initial Database)

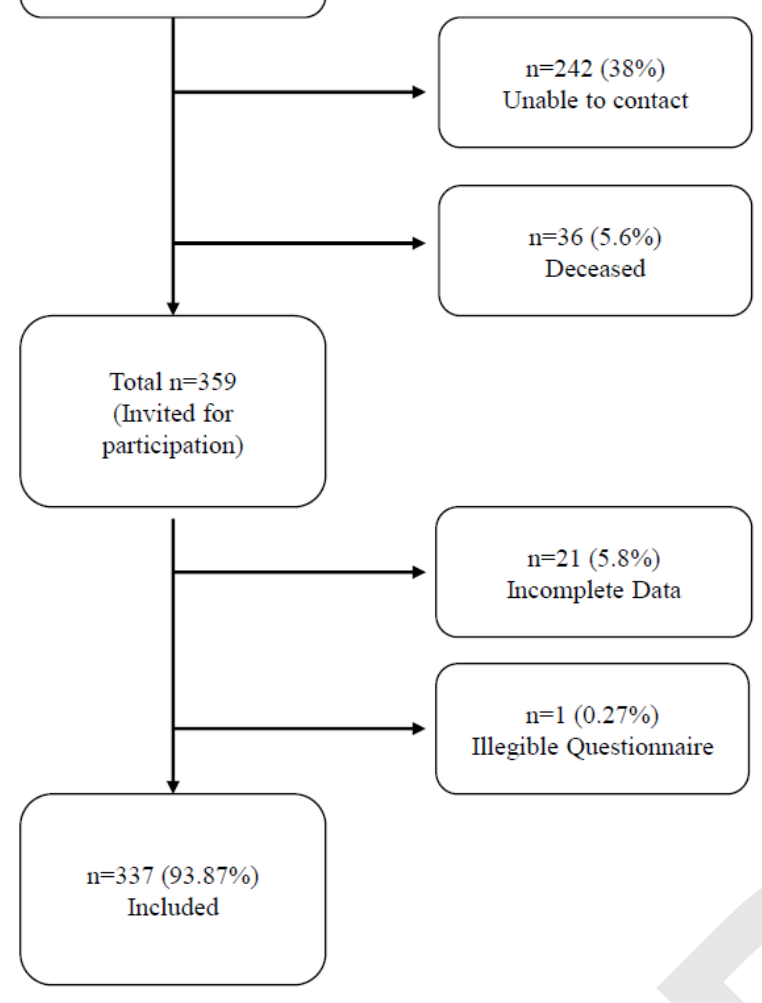

Figure 1: Patient inclusion flowchart: Initially 637 patients were included in the primary study population. Of these, $242(38 \%)$ patients could not be contacted and $36(5.6 \%)$ patients died. Of 359 patients invited to participate, $21(5.85 \%)$ patients returned an incomplete questionnaire and one $(0.27 \%)$ patient returned an illegible questionnaire that could not be evaluated, leaving $337(93.87 \%)$ patients who were enrolled in this study. $n=$ number 


\begin{tabular}{lc}
\hline \multicolumn{2}{c}{ Demographics } \\
\hline Age (years) & $25.44( \pm 11.69)$ \\
Gender (male) & $223(66.0 \%)$ \\
Married (yes) & $107(31.7 \%)$ \\
ISS & $20.3( \pm 9.264)$ \\
MAIS 1 Head & $2.68( \pm 0.77)$ \\
MAIS 2 Face & $1.61( \pm 0.71)$ \\
MAIS 4 Thorax & $3.23( \pm 1.17)$ \\
MAIS 5 Abdomen & $2.58( \pm 0.72)$ \\
MAIS 6 Spine & $2.54( \pm 1.14)$ \\
MAIS 7 UpperEx & $2.13( \pm 0.56)$ \\
MAIS 8 LowerEx & $2.77( \pm 0.46)$ \\
\hline
\end{tabular}

Table 1: Demographics of patients who were included in this article. Means ( \pm Standard deviation) for each demographic are shown. PTSD = Post-traumatic Stress Disorder, ISS = Injury Severity Score, MAIS = Maximum Abbreviated Injury Scale, ns = not significant 


\begin{tabular}{|c|c|c|c|c|c|c|c|c|c|c|c|}
\hline & \multirow{3}{*}{$\begin{array}{c}\text { Total } \\
\mathrm{n}=337\end{array}$} & \multicolumn{8}{|c|}{ PTSD-related symptoms } & \multicolumn{2}{|c|}{$\begin{array}{l}\text { All PTSD-related } \\
\text { symptoms }\end{array}$} \\
\hline & & \multicolumn{2}{|c|}{ None } & \multicolumn{2}{|c|}{ Intrusion } & \multicolumn{2}{|c|}{ Avoidance } & \multicolumn{2}{|c|}{ Hyperarousal } & \multicolumn{2}{|c|}{ PTSD } \\
\hline & & $\mathrm{n}=178$ & $\mathrm{p}$-value & $\mathrm{n}=65$ & $\mathrm{p}$-value & $\mathrm{n}=92$ & p-value & $\mathrm{n}=95$ & $\mathrm{p}$-value & $\mathrm{n}=10$ & p-value \\
\hline Psychiatric Treatment after Trauma & $71(21.0 \%)$ & $46(25.8 \%)$ & 0.01 & $13(20.0 \%)$ & ns & $9(9.8 \%)$ & 0.001 & $7(7.4 \%)$ & $<0.001$ & $5(50 \%)$ & 0.01 \\
\hline $\begin{array}{l}\text { Psychiatric Treatment before } \\
\text { Trauma }\end{array}$ & $6(1.8 \%)$ & $3(1.7 \%)$ & ns & $2(3.1 \%)$ & ns & $2(2.2 \%)$ & ns & $2(2.1 \%)$ & ns & $1(10 \%)$ & ns \\
\hline Inpatient psychiatric Treatment & $27(8.0 \%)$ & $16(9.0 \%)$ & ns & $4(6.2 \%)$ & $\mathrm{ns}$ & $2(2.2 \%)$ & 0.01 & $2(2.1 \%)$ & 0.01 & $4(40 \%)$ & $<0.001$ \\
\hline Retirement due to Trauma & $113(33.4 \%)$ & $62(34.8 \%)$ & ns & $21(32.3 \%)$ & ns & $27(29.3 \%)$ & ns & $28(29.5 \%)$ & ns & $4(40 \%)$ & ns \\
\hline Unemployment due to Trauma & $49(14.5 \%)$ & $30(16.9 \%)$ & ns & $6(9.2 \%)$ & ns & $11(12.0 \%)$ & ns & $8(8.4 \%)$ & 0.04 & $5(50 \%)$ & 0.009 \\
\hline $\begin{array}{l}\text { Additional Psychiatric Insults after } \\
\text { Trauma }\end{array}$ & $88(26.1 \%)$ & $49(27.5 \%)$ & ns & $14(21.5 \%)$ & ns & $19(20.7 \%)$ & ns & $18(18.9 \%)$ & ns & $5(50 \%)$ & ns \\
\hline
\end{tabular}

Table 2: Psychiatric covariates of patients who suffered from PTSD and either no, some or all PTSD-related symptoms at least once a month. Numbers (\%) of positive responses for each covariate are shown. Covariate numbers in each patient group were compared to asymptomatic patients who did not report the respective covariate and PTSD-related symptom. PTSD $=$ Posttraumatic Stress Disorder, ns = not significant. 